\title{
Parallax measurements of the Mira-type star UX Cygni with phase-referenced VLBI
}

\section{Tomoharu Kurayama*}

Mizusawa VERA Observatory, National Astronomical Observatory of Japan

E-mail: kuraymtm@cc.nao.ac.jp

\begin{abstract}
We have measured the annual parallax of the Mira-type star UX Cygni of $0.54 \pm 0.06$ mas using phase-referenced VLBI observations of its circumstellar water masers with the VLBA. The corresponding distance is $1.85_{-0.18}^{+0.25} \mathrm{kpc}$. This is the first measurement of star's annual parallax based on the observations of water masers. Some kinds of variable stars, including Mira variables, have period-luminosity relations, and so they are used as distance indicators. However, they are only relative distance indicators because we do not know the relationships between periods and absolute luminosities. If we could measure this relationship for Mira variables, it would become an absolute distance indicator, allowing for an independent measurement of the distance to Large Magellanic Cloud (LMC), which is important for the calibration of period-luminosity relations for other kinds of variable stars. Our result is consistent with the period-luminosity relation of Mira variables in LMC and its distance from present observations.
\end{abstract}

The 8th European VLBI Network Symposium

September 26-29, 2006

Toruń, Poland

\footnotetext{
*Speaker.
} 


\section{Introduction}

It is well known that there exist period-luminosity relations for some kinds of variable stars such as Cepheids and RR Lyrae. These relationships between the variation periods and magnitudes are used for distance estimations of near galaxies as parts of "distance ladders". However, such relations are not established well enough for any type of variables. Thus, they are relative distance indicators now as we can estimate only the ratio of distances by comparing the period-luminosity relations of two groups.

The period-luminosity relation for Miras has been studied using Miras in Large Magellanic Cloud (LMC). For example, [1] shows the period-luminosity relation for 55 Miras in LMC. Especially, the $K$-band relation has a weak dependence on chemical composition of Miras and so it is potentially useful for determining distances. However, the distance to LMC is not established sufficiently well, so we cannot establish the relation between variation periods and absolute magnitudes for LMC Miras. Therefore, we need to establish it based on determinations of distances to Galactic Miras. Unfortunately, period-luminosity relation of solar-neighbourhood Miras is not well established compared to for LMC Miras. Even with Hipparcos results, which are the best measurements of parallaxes to date, the errors from distance uncertainties are still large for those Miras [2, 目, 舟. In [2], 16 Miras were used for finding period-luminosity relation. Among them, parallaxes of only 7 Miras satisfy $\varpi / \varepsilon(\varpi) \geq 3.0$ and $\varepsilon(\varpi)<1.75$ mas (where $\varpi$ is annual parallax, $\varepsilon(\varpi)$ is the error of $\varpi)$ [5, 6 ]. The accuracy of Hipparcos' parallaxes is, thus, not enough to measure the distances to Miras.

In phase-referenced VLBI, the target source and a phase-reference source are observed simultaneously or within short intervals (see [7, pp.476-480], [8] and [9]) to make it possible to remove the effect of fluctuations of the Earth's atmosphere and use VLBI differential phase. We can also get a nearly absolute position of the target by choosing a distant source as a phase reference. Therefore, phase-referenced observations are useful for detections of weak sources and precise astrometric measurements. Among them, measures of annual parallaxes, which are direct distance measurements without assumptions, are often performed for pulsars [10, 11, 12]. $\mathrm{OH}$ maser observations are performed at $1.6 \mathrm{GHz}$ for AGB stars, too [13]. These measurements attain parallax accuracy of about 1 mas. This accuracy is still not enough to measure the distances to Miras because most of their parallaxes are of 1 mas or smaller. However, with water masers observed at $22 \mathrm{GHz}$, positional accuracies of the order of 0.05 mas can, in principle, be achieved [14]. One of the reasons for this is that the synthesized beam width at $22 \mathrm{GHz}$ is about $1 / 14$ of that at 1.6 GHz. Moreover, water masers are emitted at about $100 \mathrm{AU}$ away from Miras, while $\mathrm{OH}$ masers are at $1000 \mathrm{AU}$. Thus, we can trace stellar movements much better with water masers than $\mathrm{OH}$ masers.

For this purpose, we selected five Miras which have bright water masers and bright reference VLBI sources within 2 degrees with evenly distributed variation periods and declinations larger than $+10^{\circ}$, so that they can be observed at high enough elevation to minimize the airmass correction effect. In this paper, we show the results for UX Cygni, which has the longest period (565 days) among the five [15]. The shapes of period-luminosity relation of Miras in LMC and in the Galaxy are different in the range of variation period from 400 to 800 days [1, 16, 17]. Thus, it is worthwhile to measure the distance of a long-period Mira UX Cygni.

In order to check the period-luminosity relation of solar-neighbourhood Miras, we have mea- 
sured the annual parallaxes of water masers in the gas around them with phase-referenced VLBI. This is the first measurement of a parallax using water masers.

\section{Observation and analysis}

The positions of 22-GHz water masers around UX Cygni were monitored over a period of almost one year with 10 stations of the NRAO VLBA ${ }^{1}$. Five-hour-long observations were performed over four epochs: 22 Feb 2001, 12 Oct 2001, 11 Nov 2001 and 4 Feb 2002. The antenna-nodding cycle was 40 seconds, in which UX Cygni was observed for about 7 seconds and phase-reference source was also observed for about 7 seconds.

The average of K-band magnitudes is 1.96 [18, 19]. The phase-reference source is $\mathrm{J} 2050+3127$, whose redshift is $z=3.18$ [20]. Separation angle between UX Cygni and the phase-reference source is 1.038 . The synthesized beam width is 0.8 mas in major-axis direction and 0.4 mas in minor-axis direction. Position angle of the major axis of the synthesized beam is $175^{\circ}$.

\section{Results}

\subsection{Water masers around UX Cygni}

Figure 17a shows the distributions of maser spots in four epochs. Map origins are at the same positions locked to the phase-reference source $\mathrm{J} 2050+3127$. The loop structure to the northeast of the star is consistent with [21].

\subsection{Fitting with a parallax and proper motions}

Over four epochs of the observations, seven maser spots were detected in all epochs. Figure $1 \mathrm{~b}$ shows their movements.

Assuming that the movements of maser spots are the sums of linear motions (proper motions) and the parallax motion on the celestial sphere, we fit them with measured right ascensions and declinations of maser spots in each epoch. Thus, theoretical equations are follows:

$$
\begin{aligned}
\Delta \alpha^{(i)} \cos \delta & =\varpi(-\sin \alpha \cos \odot+\cos \varepsilon \cos \alpha \sin \odot)+\left(\mu_{\alpha}^{(i)} \cos \delta\right) t \\
\Delta \delta^{(i)} & =\varpi(\sin \varepsilon \cos \delta \sin \odot-\cos \alpha \sin \delta \cos \odot-\cos \varepsilon \sin \alpha \sin \delta \sin \odot)+\mu_{\delta}^{(i)} t
\end{aligned}
$$

where $\left(\Delta \alpha^{(i)}, \Delta \delta^{(i)}\right)$ are the displacements of observed positions of $i$-th maser spot (positions with the parallax and proper motions minus positions without the parallax and proper motions), $\left(\mu_{\alpha}^{(i)}, \mu_{\delta}^{(i)}\right)$ are the linear motions of $i$-th maser spot (sum of inner motion and the proper motion of UX Cygni itself), $t$ is time, $\varpi$ is annual parallax, $(\alpha, \delta)$ are right ascension and declination of source, $\odot$ is ecliptic longitude of the sun, and $\varepsilon$ is obliquity of the ecliptic [22].

The result of fitting is $\varpi=0.54 \pm 0.06$ mas, $\left(\left\langle\mu_{\alpha}^{(i)} \cos \delta\right\rangle,\left\langle\mu_{\delta}^{(i)}\right\rangle\right)=(-6.91,-12.52)$ mas, where \langle\rangle denotes the average. The linear motions of maser spots $\left(\mu_{\alpha}^{(i)}, \mu_{\delta}^{(i)}\right)$ are listed in table 1. The distance to UX Cygni is $1.85_{-0.19}^{+0.25} \mathrm{kpc}$. We dropped the third-epoch data for maser spot

\footnotetext{
${ }^{1}$ The National Radio Astronomy Observatory is a facility of the Natural Science Foundation operated under cooperative agreement by Associated Universities, Inc.
} 
(a)

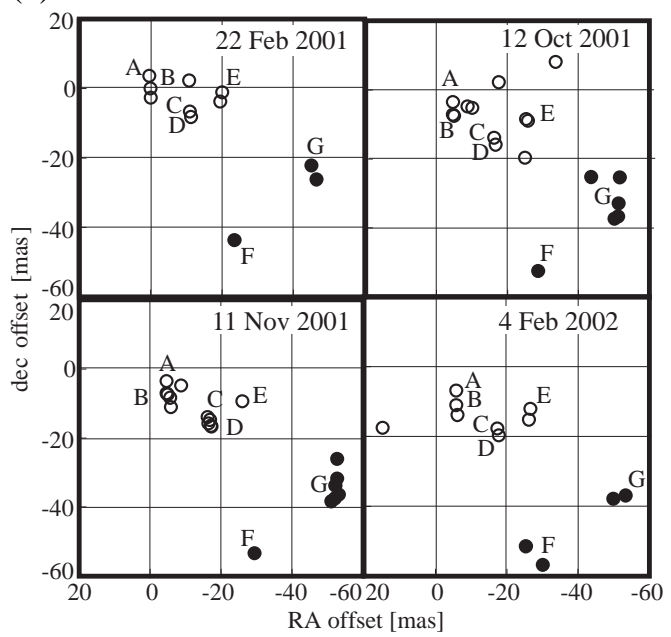

(b)

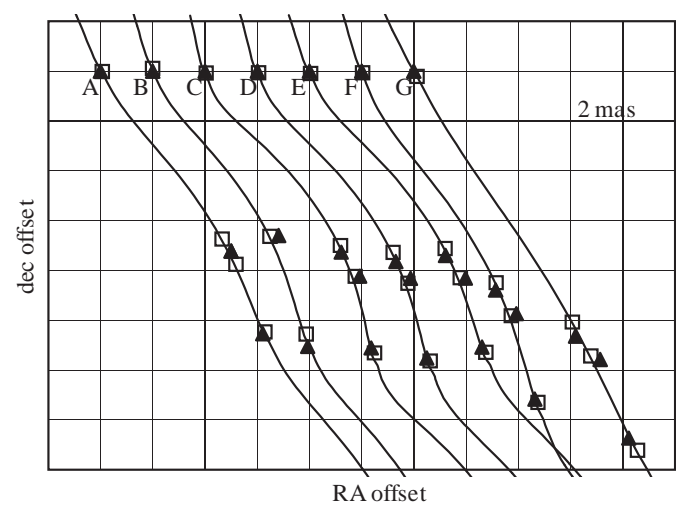

Figure 1: (a) Distribution of maser spots in each observation. Open circles are spots whose radial velocities are negative, and filled circles are positive. The radial velocity of UX Cygni itself is estimated to be -0.2 $\mathrm{km} / \mathrm{s}$ [21]. (b) Movements of maser spots and fitting results. Closed triangles show the observed positions, solid lines show the fitting results. Open squares show the theoretical positions on observed dates. One cell is 2 mas. For each maser spot, observed position for the first date is set to the intersection of division lines.

\begin{tabular}{cccc}
\hline $\begin{array}{c}\text { Maser } \\
\text { ID }\end{array}$ & $\begin{array}{c}\mu_{\alpha}^{(i)} \cos \delta \\
\text { mas/yr }\end{array}$ & $\begin{array}{c}\mu_{\delta}^{(i)} \\
\text { mas/yr }\end{array}$ & $\begin{array}{c}v_{\mathrm{LSR}} \\
\mathrm{km} / \mathrm{s}\end{array}$ \\
\hline $\mathrm{A}$ & $-6.32 \pm 0.29$ & $-10.96 \pm 0.28$ & -2.0 \\
$\mathrm{~B}$ & $-6.09 \pm 0.29$ & $-11.61 \pm 0.29$ & -2.4 \\
$\mathrm{C}$ & $-6.73 \pm 0.29$ & $-11.69 \pm 0.28$ & -4.0 \\
$\mathrm{D}$ & $-6.82 \pm 0.29$ & $-12.06 \pm 0.28$ & -3.9 \\
$\mathrm{E}$ & $-6.92 \pm 0.29$ & $-11.70 \pm 0.28$ & -2.9 \\
$\mathrm{~F}$ & $-6.92 \pm 0.29$ & $-13.85 \pm 0.28$ & 2.2 \\
$\mathrm{G}$ & $-8.59 \pm 0.29$ & $-15.79 \pm 0.28$ & 2.8 \\
\hline average & $-6.91 \pm 0.75$ & $-12.52 \pm 1.57$ & \\
\hline
\end{tabular}

Table 1: Fitting results of linear motions. Annual parallax $\varpi=0.54 \pm 0.06$ mas. $v_{\text {LSR }}$ is an average of radial velocities over time. Maser IDs (A-G) are corresponding to all figures. Errors in average columns are standard deviations of seven spots.

B because its residual was much larger than the others ( $\sim 1$ mas). Hereafter, we assume that the average value is the proper motion of UX Cygni itself. Since we assume the inner motions of maser spots to be linear motions, this method is unable to separate the inner motions from the proper motion of UX Cygni itself, but it is able to separate the parallax from the other motions. Because of the effect of inner motions, $\left(\mu_{\alpha}^{(i)}, \mu_{\delta}^{(i)}\right)$ are different. 


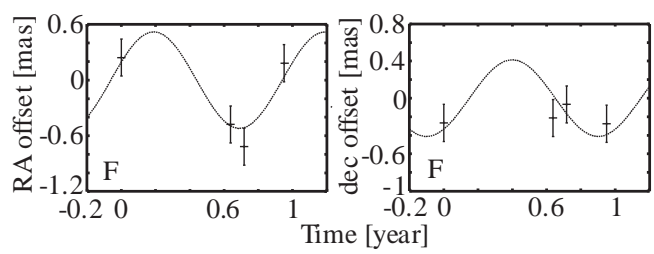

Figure 2: Right ascention or declination versus time plot for maser F. Crosses and error bars are observed data minus fitted linear motions. Dashed curves are the fitted parallax motions.

\section{Discussion}

\subsection{Comparison with the period-luminosity relation in LMC}

According to [1], the period-luminosity relation in LMC shows a bending at 420-day period. From the data given in [1], the period-luminosity relation over 420 days is:

$$
K=-5.22 \log P+23.7,
$$

where $K$ is $K$-band magnitude and $P$ is variation period [day]. From this relation, our result $\varpi=0.54 \pm 0.06$ mas and $K=1.96$, the distance to LMC is $55.1_{-7.2}^{+9.0} \mathrm{kpc}$ (its distance modulus is $18.71_{-0.31}^{+0.33}$ ). The error in distance modulus consists of $0.24-0.27$ mas from parallax error and 0.19 mas from the intrinsic width of the period-luminosity relation. This value is consistent with $50.1 \pm 0.5 \mathrm{kpc}$ given by [23]. The period-luminosity relation for galactic OH/IR stars (they are a kind of Miras) ranging between 400 and 800 days is lower than for other range of periods [16, 17], and the relation for LMC Miras is higher. This difference of shape of period-luminosity relation affects the central value $(55.1 \mathrm{kpc})$ of our results.

\subsection{Three-dimensional position and kinematics of UX Cygni in the Galaxy}

From our result ( $\varpi=0.54 \pm 0.06$ mas) we can calculate the position of UX Cygni in the Galaxy. If we assume that the Sun lies on the galactic plane and $R_{0}=8.5 \mathrm{kpc}$, the cylindrical coordinates of UX Cygni are $(R, \theta, z)=\left(8.20 \pm 0.01 \mathrm{kpc}, 12 .{ }^{\circ} 4_{-1 .{ }^{\circ} 3}^{+1 .{ }^{7}},-0.30_{-0.04}^{+0.03} \mathrm{kpc}\right)$, where the Sun lies at $(R, \theta, z)=\left(R_{0}, 0,0\right)$ and the origin is at the Galactic centre.

Using our proper motion figure $\left(\mu_{\alpha} \cos \delta, \mu_{\delta}\right)=(-6.91 \pm 0.75,-12.52 \pm 1.57)$ mas/yr, we can estimate three-dimensional velocity of UX Cygni in the Galaxy. The three-dimensional velocity of UX Cygni is $\left(V_{R}, V_{\theta}, V_{z}\right)=\left(-81.6_{-15.3}^{+14.0}, 204.0_{-3.4}^{+3.6},-12.1_{-10.3}^{+10.2}\right) \mathrm{km} / \mathrm{s}$. These values are calculated assuming $R_{0}=8.5 \mathrm{kpc}, \Theta_{0}=220 \mathrm{~km} / \mathrm{s}$ and standard solar motion. We use the converting matrix from equatorial coordinates to galactic coordinates [24] in order to convert the proper motion in equatorial coordinates to that in galactic coordinates. We used the average of maser spots $(-1.46 \pm 2.60 \mathrm{~km} / \mathrm{s})$ for the radial velocity. Errors are estimated by root sum square of each error $\left(\varpi,\left\langle\mu_{\alpha}^{(i)}\right\rangle,\left\langle\mu_{\delta}^{(i)}\right\rangle\right.$ and radial velocity).

\section{Conclusion}

We observed water masers around a Mira-type star UX Cygni with phase-referenced VLBI observations by using an extragalactic source as a phase-reference. We obtain the parallax $\varpi=$ 
$0.54 \pm 0.06$ mas. Combining the period-luminosity relation in LMC, it shows a slightly larger value of the distance to LMC, but it is affected by the displacement of the period-luminosity relation. We can get the three-dimensional position and motion in the Galaxy from the parallax and the proper motion.

\section{References}

[1] Feast, M. W., Glass, I. S., Whitelock, P. A., and Catchpole, R. M. 1989, MNRAS, 241, 375

[2] van Leeuwen, F., Feast, M. W., Whitelock, P. A., and Yudin, B. 1997, MNRAS, 287, 955

[3] Whitelock, P. and Feast, M. 2000, MNRAS, 319, 759

[4] Knapp, G. R., Pourbaix, D., Platais, I. and Jorissen, A. 2003, A\&A, 403, 993

[5] Willson, L. A. 2000, ARA\&A, 38, 573

[6] Wallerstein, G. and Knapp, G. R. 1998, ARA\&A, 36, 369

[7] Thompson, A. R., Moran, J. M. and Swenson, G. R., Jr. 2001, Interferometry and Synthesis in Radio Astronomy (2nd ed.; New York; Wiley)

[8] Alef, W. 1989, in Very Long Baseline Interferometry Techniques and Applications, ed. M. Felli and R. E. Spencer (NATO ASI Ser. C, 283; Dordrecht; Kluwer), 261

[9] Beasley, A. J. and Conway, J. E. 1995, in ASP Conf. Ser. 82, Very Long Baseline Interferometry and the VLBA, ed. J. A. Zensus, P. J. Diamond and P. J. Napier (San Francisco; ASP), 327

[10] Chatterjee, S., Cordes, J. M., Vlemmings, W. H. T., Arzoumanian, Z., Goss, W. M. and Lazio, T. J. W. 2004, ApJ, 604, 339

[11] Dodson, R., Legge, D., Reynolds, J. E. and McCulloch, P. M. 2003, ApJ, 596, 1137

[12] Brisken, W. F., Benson, J. M., Goss, W. M. and Thorsett, S. E. 2002, ApJ, 571, 906

[13] Vlemmings, W. H. T., van Langevelde, H. J., Diamond, P. J., Habig, H. J. and Schilizzi, R. T. 2003, A\&A, 407, 213

[14] van Langevelde, H. J. and Vlemmings, W. H. T. 2003, in Mass-Losing Pulsating Stars and Their Circumstellar Matter, ed. Y. Nakada, M. Honma and M. Seki (Dordrecht: Kluwer), 381

[15] Samus, N. N. et al. 2003, Astron. Lett., 29, 468

[16] Whitelock, P., Feast, M. and Catchpole, R. 1991, MNRAS, 248, 276

[17] van Langevelde, H. J., van der Heiden, R. and van Schooneveld, C. 1990, A\&A, 239, 193

[18] Feast, M. W. and Whitelock, P. A. 2000, MNRAS, 317, 460

[19] Gezari, D. Y., Pitts, P. S. and Schmitz, M. 1999, Catalog of Infrared Observations (5th ed.; Strasbourg; CDS)

[20] Fey, A. L., Boboltz, D. A., Gaume, R. A., Eubanks, T. M. and Johnston, K. J. 2001, AJ, 121, 1741

[21] Bowers, P. F. and Johnston, K. J. 1994, ApJS, 92, 189

[22] Green R. M. 1985, Spherical Astronomy (Cambridge; Cambridge Univ. Press)

[23] Alves, D. R. 2004, New A Rev., 48, 659

[24] Murray, C. A. 1989, A\&A, 218, 325 